\title{
Epidemiology of COVID-19 in Misrata, Libya: A Population-Based Surveillance Study
}

\author{
Anas Zarmouh1', Hussien Elaswdi ${ }^{*}$, Essam Elakhtel2 ${ }^{2}$ Khalid Abufalgha ${ }^{1,2}$, Mohamed Taraina ${ }^{1,2}$ \\ ${ }^{1}$ Misurata University Faculty of Medicine, Misrata, Libya \\ ${ }^{2}$ Misurata Medical Centre, Misrata, Libya \\ Email: ^Hussienelaswdi@gmail.com
}

How to cite this paper: Zarmouh, A., Elaswdi, H., Elakhtel, E., Abufalgha, K. and Taraina, M. (2021) Epidemiology of COVID19 in Misrata, Libya: A Population-Based Surveillance Study. Open Journal of Epidemiology, 11, 101-112.

https://doi.org/10.4236/ojepi.2021.111010

Received: December 16, 2020

Accepted: February 16, 2021

Published: February 19, 2021

Copyright $\odot 2021$ by author(s) and Scientific Research Publishing Inc. This work is licensed under the Creative Commons Attribution International License (CC BY 4.0).

http://creativecommons.org/licenses/by/4.0/

(c) (i) Open Access

\begin{abstract}
Background: The prevalence of SARS-COV2 in Libya is unclear; PCR is still under-performed. Although lockdown measures have been in place for weeks, there is no prevalence data to support the gradual lifting of such lockdowns. SARS-COV2 IgM/IgG is emerging as potential alternatives to PCR testing, and many counties and towns have undertaken such seroprevalence studies. Objective: Prevalence of SARS-COV2 in Misrata, Libya amongst the population as a whole. Materials and Methods: The study was prospective, cross-sectional, population-based, and age-stratified, to assess SARS-COV2 $\operatorname{IgM} / \operatorname{IgG}$ in the population of Misrata. It was carried out between the 18th and 21st of April, 2020. Results: A representative sample of 897 participants was tested for SARS-COV2 IgM/IgG antibodies. 30 participants were positive for IgM or IgG indicating a prevalence of $3.4 \%$ (95\% CI, $2.8 \%-5.2 \%)$, with eight (0.9\%) IgM positive and 22 (2.4\%) IgG positive. Accordingly, 9075 (95\% CI, 6586 - 14,170) people are estimated to have had a current or recent COVID-19 infection, symptomatic or asymptomatic. Conclusion and Recommendations: Serological testing as a diagnostic or surveillance tool may uncover a large proportion of asymptomatic individuals in the community, far outnumbering PCR. The authors highly recommend further precautionary public measures, as well as an ongoing surveillance programme to monitor the epidemiological trends of the disease in the city of Misrata and Libya in general.
\end{abstract}

\section{Keywords}

Prevalence, SARS-COV2 IgM/IgG, PCR

\section{Introduction}

COVID-19 declared as a pandemic by the World Health Organization (WHO) 
on January 13th, 2020, has been rather hesitant to invade the Libyan lands, especially, when one looks at neighbouring Tunisia, Egypt, and Italy and the aviation traffic with Turkey and China. Libya, to date, has only diagnosed fewer than 70 cases with three fatalities due to COVID-19.

The local knowledge deficit of COVID-19 epidemiology is huge; the weak government is unable to lead on large-scale mass screening programmes, which other countries have embarked on. It is, therefore, essential to establish an epidemiological landmark study in Libya to underpin further central efforts to tackle the pandemic and inform any future decisions easing up lockdowns in the cities. As PCR testing is scarce in Libya, an alternative is to measure serological markers for COVID-19 (SARS-COV2 IgM/IgG). These have become commercially available only in February 2020, and available only in February 2020. Despite validity concerns, they are considered a useful diagnostic tool in clinical practice, whilst PCR confirmation is awaited [1].

\subsection{Evidence of Epidemiological Studies into COVID-19}

The calculation of Case-Fatality Rates (CFR) and Basic Reproductive Rates (BRR) rely on the total number of infections, a number that is yet to be realistically estimated. Serological studies may provide more solid prevalence rates, than those scored by PCR testing [2].

Several governments and institutes have tackled the important question of how widespread COVID-19 truly is. Both molecular and serological testing methods have been utilised, and local resources have driven many. As asymptomatic carriers are an important route of transmission, it is even more crucial to estimate and monitor epidemiological trends of COVID-19 [3].

\subsection{Mass Testing}

COVID-19 surveillance programmes [4] \{Kolifarhood, 2020 \#24\} \{Kolifarhood, 2020 \#24@@author-year\} have taken off in several countries including the USA, UK, Germany, South Korea, and others. Surveillance outcomes, according to those countries, will inform and guide public health management decisions of the pandemic, including the gradual lifting of city lockdowns and other public activities [5].

The American CDC lists the following as goals for COVID-19 national surveillance $\{$ Prevention, 2020 \#26\} [6]:

- To monitor the spread and intensity of COVID-19 disease in the United States;

- To understand disease severity and the spectrum of illness;

- To understand risk factors for severe disease and transmission;

- To monitor for changes in the virus that causes COVID-19;

- To estimate disease burden;

- To produce data for forecasting COVID-19 spread and impact;

- To understand how COVID-19 impacts the capacity of the U.S. healthcare system (e.g., availability and shortages of key resources). 
South Korea has adopted mass testing very early and has, in addition to other important factors, succeeded in containing the epidemic with and over 11,000 tests per million population with a surge in cases lasting less than 20 days (24/02/ 2020 to $09 / 03 / 2020$ ) and only 244 deaths [7]. An ambitious suggestion to weekly PCR-test the whole UK population to end the epidemic was published in the BMJ [8]. The UK is now testing over 100,000 per day, some via drive-through sites to build up enough epidemiological data to guide lockdown decisions [9].

The Iceland population study, published by NEJM, PCR -screened $6 \%$ of the population, they used three methods of recruitment and yielded varying degrees of prevalence. 1) Targeted population at two subsequent time intervals with infection rates at $9.2 \%$ and $14.4 \%$ of 1924 and 7275 at-risk persons, respectively; 2) Populations screening at two-time intervals also reported $0.8 \%$ and $0.6 \%$ of 10,797 and 2283 randomly selected persons in Iceland. The study, although, PCR based, demonstrates the clear contrast when screening at-risk populations from screening the general population [10].

\subsection{Evidence Supporting the Use of Serological COVID-19 Markers}

Early in this pandemic, several commercial firms developed serological testing for SARS-COV2 IgM and IgG. Quantitative tests use ELISA (enzyme -linked immunosorbent assay) or CLIA (Chemiluminescence Immunoassay), whilst qualitative tests (also known as Rapid Diagnostic Tests-RDT) use lateral flow immunoassay to estimate IgM and IgG antibodies. However, the WHO maintains that SARS-CoV-2 confirmation by PCR remains the gold standard for case confirmation, reporting, and notification [9] [11]. Timeline studies have demonstrated the relationship between COVID-19 symptoms and IgM to be at 3 days and IgG to be 14 days after onset of symptoms [11] [12].

\subsection{Seroprevalence Studies}

There have been several survey studies to stand at the prevalence of SARS-COV2 IgM/IgG amongst whole populations. This is in contrast to validation studies to test the performance of the immunoassay against NAAT-confirmed cases yielding sensitivity rates of up to $96 \%$ and specificity of $95 \%$ [12] [13].

Preprint work by an international group from the USA, Finland, and Switzerland described how a serological test was developed, but more importantly, draws attention to three important concepts, 1) the potential application of serological testing in mass population screening; 2) defining previous exposure in individuals and determine immunity to return/resuming work 3) convalescent plasma donations [14]. This importance is also re -iterated by Johns Hopkins Centre for Health Security [15].

The Council of State and Territorial Epidemiologists (CSTE) [1] considers COVID-19 serological markers (IgM/IgG) to be amongst criteria for reporting COVID-19 cases, but in the presence of clinical features consistent with the condition, this in contrast to the gold standard of virus detection using Nucleic Acid 
Amplification Testing (NAAT) such as PCR. The WHO's position on reporting still considers PCR as the gold standard for reporting the disease, however, the WHO's director-general briefing on 03/03/2020 did mention antibody screening for populations to be a forward option and he encouraged countries to pursue this avenue [16].

The Santa Clara county seroprevalence study, still under preprint, tested 3300 people in California, USA, and reported the population -weighted prevalence $2.81 \%$ (95\% CI, 2.24 - 3.37) [17]. The authors imply that serological testing is much more reliable than the gold standard PCR testing, as it can detect immunity in asymptomatic and mildly symptomatic persons, whilst PCR performance in such populations is likely to be falsely low. Furthermore, another unpublished study by the University of Southern California, declared in a press conference on 20/04/2020, 863 representative samples of Los Angeles country (adult population of $8 \mathrm{~m}$ ) and $4.1 \%$ tested positive. They estimated 320,000 prior infection by COVID-19. This gives it a magnitude of 40 times the PCR confirmed in the county of LA [18]. In Italy, there were two large serological surveys; the first was conducted in the town of Robbio, where all inhabitants were tested (6000) with a reported prevalence of $11 \%$. The second town to be tested in whole was Vo of 3000 inhabitants and reporting a contrasting 3\% prevalence. Despite the high media coverage of the two studies, there has been no publication since their completion in March 2020 [19] [20].

In the German town of Gangelt, 1000 serology samples reported an infection rate of $14 \%$ with $2 \%$ being concurrently PCR positive. Although, selection bias hampers any serious conclusions from this unpublished study, but it does signal the wide non-concordance between NAT and serological testing [21].

More lately, another study in Guilan province, Iran reported a crude seroprevalence rate as high as $22 \%$, after testing 550 subjects and concluded that infection rates in the province were between 55 and 77 thousand people [22]. Furthermore, the latest to be pre-published was a study in Japan of 1000 serum samples from Kobe, which found 33 positive IgG samples [23].

Many of those studies lack peer review, however, almost all have been completed in the past 6 weeks, and therefore, may not be ready yet. It is also clear that such seroprevalence studies have innate bias and weaknesses related to sampling, recruitment, and test validity. However, it is imperative to include such results in the public health decision-making processes, as the price of ignoring the evidence is too high.

\subsection{Objectives}

The main aim of this study is to provide governmental bodies and public health institutes with information to support forward decision-making with regards to city lockdown, curfews, and other precautionary measures, which have been put in place since March 2020.

The objectives of the study are:

- To estimate the current disease burden of COVID-19 in Misrata, by detecting 
SARS-CoV2 IgM/IgG antibodies in the city;

- To delineate disease prevalence in various socio -demographic characteristics.

\section{Methods}

This was a cross -sectional survey study, looking at the epidemiology of COVID-19 in Misrata. It was carried out between the 18th and 21st of April, 2020. Similar studies have been termed "Seroprevalence studies". A representative sample was screened for quantitative IgM/IgG antibodies against SARS-CoV2, using CLIA technique (Maglumi 2000 plus, Snibe) amongst adults aged 18 years and above. The WHO has issued guidance conducting such studies, [24] this guidance was followed and results will be communicated to them, as the organization is building a multi -national database to study the prevalence of disease as well as proportion of symptomatic patients to asymptomatic.

\subsection{Sample Size Calculation}

The calculation was undertaken using Epi-info software version 7, based on 50\% prevalence of infection (current or previous) and a confidence limit of 5\%, the minimum required sample size of $95 \%$ confidence level is 384 participants. A decision was made to increase the sample size to 1000 participants to minimise sampling error.

\subsection{Sampling Technique and Participant Selection}

The prospective population based used convenience sampling, stratified by age. Misrata, being the third most populous city in Libya with 550,000 inhabitants, has an area of $5573 \mathrm{~km}^{2}$ [25]. The civil registration system divides the city into 10 communities. The research team was unable to obtain up to date detailing inhabitants with age distributions, but community populations were secured from the 2019 registrar's office data. Therefore, based on the 2012 consensus, age distributions were drawn across the 10 communities, in the following manner:

- 40\%: Age range 18 - 39;

- 40\%: Age range 40 - 64;

- 20\%: Age range 65 and above.

Further convenience sampling strategy was based on a first-come-first-served basis after a media campaign lasting 3 days prior to the survey days and 3 days into the survey dates. The participants were selected with a probability proportional to the size of each community in the municipality.

\subsection{Data Collection Methods and Tools}

A pre-coded structured interviewing questionnaire was designed to collect sociodemographic data which include (age, gender, level of education, and address), behavioural factors, including smoking status, and brief medical history of participants. 


\subsection{Inclusion and Exclusion Criteria}

- Ages below 18 years were excluded;

- Libyan and non -Libyan residing in Misrata were included.

\subsection{Testing Procedure}

Five sites for blood sample collection were set up at local schools. There was a series of announcements on local radios, social media pages, and word of mouth to encourage participation. When participants arrived at the school site, they were requested to read and sign a consent form and a health questionnaire, after reading the provided information leaflet. Blood was drawn, under universal precautions as well as contact precautions. Sampling continued from 18/04/2020 until 21/04/2020 averaging 5 hours a day. Furthermore, two sample deliveries a day were made to "Medlab Laboratory" for processing. Samples were double code-labelled to allow for blinding both the phlebotomist and lab technician from the results. Any positive results were phoned to the chest clinicians in the research team, who arranged further PCR testing and case management.

\subsection{Test Kit Performance}

The manufacturer's supplied information included validity data detailing a combined (IgM/IgG) sensitivity of $95.6 \%$ of 91 PCR confirmed COVID-19 patients, in addition to a combined (IgM/IgG) specificity of $96 \%$ of 750 non -SARS-COV2 coronavirus samples, normal samples, and interference samples. Furthermore, 131 samples were evaluated for IgM/IgG (Maglumi) vs IgA/IgG (Euro -immun) and yielded significant agreements between the two assays [13]. Finally, the test kit and analyser have been recommended by the University of Padova, Italy, and Eurofins Scientific Group's Megalab in Spain. Locally, the Maglumi 2000 plus analyser was control tested by assessing $6 \mathrm{IgM} / \mathrm{IgG}$ assays to PCR positive patients' sera, and the assessment of 5 participants sera, who agreed to re-test, all were found to prove the reliability of the quantitative assessment. The concern of cross reactivity of such assays is founded, as a 2015 study showed coronaviruses to be responsible for $0.2 \%$ of community -acquired pneumonia population of 2320 patients [26].

\subsection{Ethical Approval}

Ethical approval was obtained from the faculty of medicine, Misrata University. It is not believed that this test or its result would expose patients to undue stress, risk, or harm as a direct or indirect effect. The researchers complied with the International Ethical Guidelines for Epidemiological Studies. An informed written consent was obtained from all participants after vast explanations of the objectives and benefits of the study and confidentiality was ensured.

\subsection{Data Analysis}

Data were computerized and descriptive statistics including frequency, distribu- 
tion, and percentage were calculated. Epi info. and Statistical Product and Service Solutions (SPSS) version 20 were used for data analysis.

\section{Results}

Over 4 days, 897 participants came forward to five pre-selected schools representing 10 communities in Misrata. A signed consent, a health-related questionnaire, and a blood sample were collected from participants. Table 1 shows the socio-demographic characteristics of all participants. The age ranged between

Table 1. Socio-demographic characteristics of all the participants.

\begin{tabular}{|c|c|c|}
\hline Socio-demographic characteristics & $\mathrm{n}=897$ & $\%$ \\
\hline \multicolumn{3}{|l|}{ Age (years) } \\
\hline $18-39$ & 337 & 37.6 \\
\hline $40-64$ & 471 & 52.5 \\
\hline $65 \geq$ & 89 & 9.9 \\
\hline \multicolumn{3}{|l|}{ Gender } \\
\hline Male & 642 & 71.6 \\
\hline Female & 255 & 28.4 \\
\hline \multicolumn{3}{|l|}{ Level of education } \\
\hline Illiterate & 52 & 5.8 \\
\hline Elementary & 186 & 20.7 \\
\hline Secondary & 259 & 28.9 \\
\hline University \& above & 400 & 44.6 \\
\hline \multicolumn{3}{|l|}{ Community residence } \\
\hline Shohada Armayla & 138 & 15.4 \\
\hline Azarook & 124 & 13.8 \\
\hline Dat Arymal & 121 & 13.5 \\
\hline Almahjob & 119 & 13.3 \\
\hline Central Misrata & 109 & 12.2 \\
\hline Tomyna & 101 & 11.2 \\
\hline Algheran & 79 & 8.8 \\
\hline Ras Altoba & 56 & 6.2 \\
\hline Gaser Ahmed & 30 & 3.3 \\
\hline Aldafnia & 20 & 2.2 \\
\hline \multicolumn{3}{|l|}{ Smoking } \\
\hline Non-smoker & 602 & 67.1 \\
\hline Smoker & 202 & 22.5 \\
\hline Former smoker & 93 & 10.4 \\
\hline \multicolumn{3}{|l|}{ Quarantine commitment } \\
\hline Poor & 70 & 7.8 \\
\hline Fair & 433 & 48.3 \\
\hline Good & 394 & 43.9 \\
\hline
\end{tabular}


18 and 92 years, with a mean $44.5 \pm 14.7$ years and a median of 44 years. More than half of the participants (52.5\%) were in the age group of 40 to less than 65 years. Nearly three -quarters of participants (71.6\%) were males. As for the education level, less than half of the participants (44.6\%) had university and above education. The least percentage was illiterate (5.8\%). More than of two-thirds of participants were non-smokers.

All 897 blood samples were tested, 30 were positive for IgM or IgG. This gives a prevalence rate of $3.34 \%$ (95\% CI, $2.8 \%$ - 5.2\%). There were eight $(0.9 \%)$ positive IgM and $22(2.44 \%)$ positive IgG. It was estimated that the population of over 18s in Misrata, according to age distributions obtained from the 2012 census and total number of inhabitants from Dec 2019, was 275,000. Accordingly, the estimated overall number of positive IgM was at 2475 (95\% CI, 2333 - 5435) and the number of people with positive IgG was at 6600 (95\% CI, 4253 - 8735). Table 2 demonstrates the seroprevalence results.

Participants whose tests were positive were contacted and further inquiry revealed two cases to be a married couple, two cases were sisters -in -law living next door to each other, and two further unrelated healthcare workers. None of the positive cases was acutely sick and therefore was reassured. All 30 positive participants were offered PCR swabs, 25 only presented for a swab, 2 were repeated and all were negative by PCR. All eight IgM positive participants were requested to home-quarantine for 14 days.

\section{Discussion}

This cross -sectional seroprevalence study is the first of its kind to be carried out in a very low prevalence community, where only 10 COVID-19 patients were PCR-diagnosed in the city of Misrata with no fatalities.

Results demonstrate a total of 30 participants with immune responses to COVID-19 out of 897 screened participants, yielding a prevalence rate of $3.44 \%$ (95\% CI, 2.39 - 5.15), and a potential 9075 (95\% CI, 6586 - 14,170) infected persons (current and past) in the city. IgM and IgG prevalence of 8 and 22 participants respectively, give a prevalence rate of $0.9 \%$ (95\% CI, $0.85-1.97)$ and $2.5 \%$ (95\% CI, 1.55 - 3.17) respectively, and a potential of 2475 (95\% CI, 2333 - 5435) and 6600 (95\% CI, 4253 - 8735) infected persons respectively.

Table 2. Results of participants who tested positive for either IgM and/or IgG.

\begin{tabular}{cccc}
\hline Test & Study sample result & $\%$ & $\begin{array}{c}\text { Estimated number of cases in } \\
\text { the whole population }\end{array}$ \\
IgM & 8 & 0.89 & 2475 \\
IgG & 22 & $(95 \%$ CI, 2333 - 5435) \\
Cumulative & 2.45 & 6600 \\
& 30 & 3.34 & $(95 \% \mathrm{CI}, 4253-8735)$ \\
& & $(95 \% \mathrm{CI}, 6586-14,170)$ \\
\hline
\end{tabular}


Whilst attempting to contrast the seroprevalence rate of $3.34 \%$ to the presumed PCR -based infection rate in Misrata of $0.002 \%$ is a serious task, this outcome is well supported in the literature, as these results of outnumbering PCR -based statistics have been reproduced in several studies with varying degrees of seroprevalence at $2.81 \%$ (Santa Clara County, US), 4.1\% (Southern Los Angeles County, US), 14\% (Gangelt, Germany), 11\% (Robbio, Italy), 3\% (Vo, Italy), 22\% (Guilan province, Iran) and 33\% (Kobe, Japan) respectively [17]-[23].

Furthermore, the study provides for strong evidence of the possible very low case fatality rate (CFR) of the SARS-COV2, given the huge asymptomatic carriage of the disease, and lack of fatality in the city. The reality of under -testing does provide for an underestimate of the true extent of disease in the city, as Libya has achieved only 71 tests per million population, at the time of the survey. Only a total of 94 swabs were taken from Misrata on a strict-indication basis.

The eight IgM seropositive cohort of asymptomatic participants tested negative for PCR. This is very likely as the quoted sensitivity for PCR testing of $63 \%$ was referenced to sick individuals, with presumed high nasopharyngeal viral load. This is contrary to our asymptomatic group, with much lower viral load in the mucosal surfaces accounting for the inability of PCR -based test to detect SARS-COV2 in the small seropositive cohort in the study. Household cross-infection amongst asymptomatic participants is still possible, as one couple tested IgG positive, two sisters -in -law and a healthcare worker.

From this study and others mentioned above, and as Table 3 summarises, it can be argued that SARS-COV2 IgM/IgG detection rates far outnumber PCR's ability to detect disease, especially the subclinical. Furthermore, serology is a more convenient and easily collected test than the nasal swab, as the latter requires certain protective precautions as well as special staff training. The low levels of IgM/IgG detected for such asymptomatic patients was also referenced in a WHO report on ID passports, suggesting that low levels of humoral immunity may indicate a further role of cellular immunity against COVID-19 [2] .

Table 3. Summarizes the various factors of serological results outnumbers PCR-based testing.

Factors causing serological results to outnumber PCR -based testing results
PCR-related-leading to underestimation of cases
Sample collection, transport, storage, and processing.
Reduced viral load in asymptomatic cases.
Focus of infection not always nasopharynx, in many cases lungs.
Serological
Immune response sensitive (when tested with confirmed cases) and specific
(when tested on pre-COVID-19 sera).
The presence of antibodies indicates current or past exposure, whereas PCR testing
may only indicate current infection.
The load threshold for antibody production may be much lower than that required
for PCR testing to be positive.


The study design allowed for population-weighting, so that the number of participants relative to its population represents each community (municipality branch). The randomization method also allowed any person to come forward to be tested, as long as the age group he/she belonged to is still open.

Limitations of the study include the male participant majority of over $70 \%$. This is inherent to the social nature of Libyan society, as males tend to be outdoors more than females, and therefore, would present themselves more readily for a test. It can be argued, however, that if a husband becomes infected, it is likely that infection will also spread to the wife and vice -versa. Participants who think they may have or have had the virus, and therefore self -selected to be tested. On the other hand, questionnaire data shows the number of people who have reported respiratory symptoms in the past 3 months to be small 196 (21.8\%).

This study reports unanticipated prevalence rates at 3.34\%, it is likely that acceptance to the public and uptake by decision-makers will be weak. The city (and the country) is entering the 8th week of lockdown and people and businesses are growing anxious about the unpredictable duration of the lockdown. Some people are calling for further extension of the restrictions; others want normal life to resume. It is, therefore, imperative that decision-makers consider very seriously the results of the study and the potential very widespread infection rate amongst people.

\section{Conclusions}

The prevalence rates of SARS-COV2 IgM/IgG antibodies in Misrata is relatively high, it far outnumbers the PCR-detected rates. This does indicate the low virulence and low case fatality rates (CFR) but also suggests the very high prevalence of disease in societies of low PCR -detected cases. The study may also contribute to establishing pools of convalescent plasma donations, once further procedural information is validated.

Further extensive validation studies are needed to establish SARS-COV2 serology tests as a reliable standard, not only for patient care but also for the wider use of population based large-scale surveillance programmes. WHO office will be notified of these results to strengthen the statistical power of such studies [24].

Mass testing is needed to discover as much of the asymptomatic carriers as possible, and uncover further portions of the hidden iceberg. Whether this testing is PCR-based or serological remains a controversy, given the apparent superiority of serology tests in detecting asymptomatic carriers.

Such seroprevalence data, along with a continuum of surveillance programme should support the government's response decision to the pandemic and inform further tightening or easing of restrictions in the Misrata and Libya in general.

What is already known on this topic?

- Serology testing for COVID-19 is an alternative to PCR testing but lacks reliability studies;

- COVID-19 screening in low incidence countries has not been extensive. 


\section{What this study adds?}

- Seroprevalence in low incidence areas can far outnumber perceived PCR-based cases;

- The case fatality rate may be extremely low, given the huge asymptomatic carriers;

- SARS-COV2 IgM/IgG quantitative assays may be used in mass screening and surveillance programmes.

\section{Acknowledgements}

The authors would like to thank all participants in the study, Faculty of Medicine in Misrata University, Misrata Medical Center, Medlabs laboratories and all technical staff, Student Union in Faculty of Medicine, Schools Regulatory Board in Misrata, and Laon Jadeed Media and Ads for their vital part in completing the study project.

\section{Conflicts of Interest}

The authors declare no competing interest.

\section{Authors' Contributions}

Anas Zarmouh: Conceptualisation, investigation, data curation, writing-original draft.

Hussien Elaswdi: Conceptualisation, investigation, data curation, project administration, formal analysis.

Essam Elakhtel: Conceptualisation, investigation, data curation, writing-review \& editing

Mohamed Taraina: Writing-review \& editing, investigation, data curation, supervision.

Khalid Abufalgha: Investigation, resources, data curation, writing-review \& editing, supervision.

\section{References}

[1] Turner, K. (2020) Standardized Surveillance Case Definition and National Notification for 2019 Novel Coronavirus Disease (COVID-19). Council of State and Territorial Epidemiologists.

[2] World Health Organization (2020) Immunity Passports in the Context of COVID-19. https://doi.org/10.15557/PiMR.2020.0024

[3] Rothan, H.A. and Byrareddy, S.N. (2020) The Epidemiology and Pathogenesis of Coronavirus Disease (COVID-19) Outbreak. Journal of Autoimmunity, 109, Article ID: 102433. https://doi.org/10.1016/j.jaut.2020.102433

[4] Kolifarhood, G., et al. (2020) Epidemiological and Clinical Aspects of COVID-19; a Narrative Review. Archives of Academic Emergency Medicine, 8, e41.

[5] Chadwick, L. (2020) Coronavirus: Antibody Tests "Will Be Crucial” in Determining When to Lift Lockdowns. EURONEWS.

[6] National Center for Disease Control and Prevention (2020) Coronavirus Disease 2019 (COVID-19)—Cases, Data \& Surveillance-Purpose and Methods. 
https://www.cdc.gov/coronavirus/2019-ncov/covid-data/covidview/purpose-metho ds.html

[7] Worldometer (2020) World/Countries/South Korea Coronavirus Cases.

[8] Peto, J. (2020) COVID-19 Mass Testing Facilities Could End the Epidemic Rapidly. BMJ, 368, m1163. https://doi.org/10.1136/bmj.m1163

[9] NHSProviders (2020) Daily Updates. https://nhsproviders.org/topics/COVID-19/coronavirus-member-support/nationalguidance/government-updates/daily-updates

[10] Gudbjartsson, D.F., et al. (2020) Spread of SARS-CoV-2 in the Icelandic Population. New England Journal of Medicine, 382, 2302-2315.

[11] World Health Organization (2020) Laboratory Testing for Coronavirus Disease (COVID-19) in Suspected Human Cases: Interim Guidance, 19 March 2020.

[12] Padoan, A., et al. (2020) Analytical Performances of a Chemiluminescence Immunoassay for SARS-CoV-2 IgM/IgG and Antibody Kinetics. Clinical Chemistry and Laboratory Medicine, 58, 1081-1088. https://doi.org/10.1515/cclm-2020-0443

[13] Lippi, G., et al. (2020) Assessment of Immune Response to SARS-CoV-2 with Fully Automated MAGLUMI 2019-nCoV IgG and IgM Chemiluminescence Immunoassays. Clinical Chemistry and Laboratory Medicine, 58, 1156-1159.

https://doi.org/10.1515/cclm-2020-0473

[14] Amanat, F., et al. (2020) A Serological Assay to Detect SARS-CoV-2 Seroconversion in Humans. Nature Medicine, 1-4.

[15] Gronvall, G., et al. (2020) Developing a National Strategy for Serology (Antibody Testing) in the United States. The Johns Hopkins Center for Health Security, 1-38.

[16] World Health Organization (2020) WHO Director-General's Opening Remarks at the Media Briefing on COVID-19.

[17] Sood, N., Simon, P., Ebner, P., Eichner, D., Reynolds, J., Bendavid, E. and Bhattacharya, J. (2020) Seroprevalence of SARS-CoV-2-Specific Antibodies among Adults in Los Angeles County, California, on April 10-11, 2020. JAMA, 323, 2425-2427. https://doi.org/10.1001/jama.2020.8279

[18] Hopper, L. (2020) Early Antibody Testing Suggests COVID-19 Infections in L.A. County Greatly Exceed Documented Cases. USC News.

[19] Andrea Crisanti, A.C. (2020) In One Italian Town, We Showed Mass Testing Could Eradicate the Coronavirus. The Guardian.

[20] Zorzoli, M. (2020) I nuovi dati di Robbio, unico paese italiano a fare il test sull'immunità a tutti i cittadini. Business Insider Italia.

[21] Streeck, H., et al. (2020) Vorläufiges Ergebnis und Schlussfolgerungen der COVID19 Case-Cluster-Study (Gemeinde Gangelt).

[22] Shakiba, M., et al. (2020) Seroprevalence of COVID-19 Virus Infection in Guilan Province, Iran. https://doi.org/10.1101/2020.04.26.20079244

[23] Doi, A., et al. (2020) Estimation of Seroprevalence of Novel Coronavirus Disease (COVID-19) Using Preserved Serum at an Outpatient Setting in Kobe, Japan: A Cross-Sectional Study. https://doi.org/10.1101/2020.04.26.20079822

[24] World Health Organization (2020) Population-Based Age-Stratified Seroepidemiological Investigation Protocol for COVID-19 Virus Infection.

[25] Misrata Municipality (2019). https://misrata.gov.ly

[26] Jain, S., et al. (2015) Community-Acquired Pneumonia Requiring Hospitalization among US Adults. New England Journal of Medicine, 373, 415-427. https://doi.org/10.1056/NEJMoa1500245 